\title{
QUANTITATIVE DETERMINATION AND PREPARATIVE ISOLATION OF TWO MAJOR ALKALOIDS FROM THE VIETNAMESE MEDICINAL HERB EVODIAE FRUCTUS
}

\section{N. VAN T. NGUYEN ${ }^{*}$, X. DAO T. NGUYEN¹, K. NGAN H. NGUYEN¹, T. KIEN NGUYEN¹, M. PHUONG NGUYEN, KYEONG-HO KIM²}

${ }^{1}$ Can Tho University of Medicine and Pharmacy, Viet Nam; 179 Nguyen Van Cu Street, Ninh Kieu District, Can Tho city, Viet Nam, ${ }^{2}$ College of Pharmacy, Kangwon National University, Chuncheon 200-701, Korea

Email: ntnvan@ctump.edu.vn

Received: 12 Jul 2019, Revised and Accepted: 30 Sep 2019

\section{ABSTRACT}

Objective: To develop a simple and accurate HPLC-DAD method for simultaneous determination, the content of major components: limonin, evodiamine, and rutaecarpine in Evodiae fructus and evaluation the quality of Evodiae fructus sold in markets.

Methods: Open column chromatography was used to separate and purify rutaecarpine and evodiamine, the two major alkaloids from Evodiae fructus extract as a laboratory standard. Chromatographic separation was achieved using a Germini $\mathrm{C}_{18}$ column $(150 \mathrm{~mm} \times 4.6 \mathrm{~mm}$ I.D., $5 \mu \mathrm{m})$, detected at $210 \mathrm{~nm}$. The mobile phase consisted of acetonitrile (A), methanol (B), and water (C). The validated method simultaneously determined alkaloid content in 40 batches of samples collected from markets in different regions of Vietnam.

Results: In one-step purification, our method yielded $326 \mathrm{mg}$ of rutaecarpine and $128 \mathrm{mg}$ of evodiamine from $3.2 \mathrm{~g}$ of crude extract, with purities of 98.9 and $98.5 \%$, respectively. The structures of these compounds were identified using ${ }^{1} \mathrm{H}$ NMR and ${ }^{13} \mathrm{C}$ NMR. There was a significant correlation between alkaloid content and fruit size, with a Spearman correlation coefficient of $>0.5(p<0.001)$, and there was a large difference in alkaloid contents between three maturity degrees of the fruit. Open-mouth fruits and fruits with average sizes of 4 to $6 \mathrm{~mm}$ had the highest alkaloid contents, whereas closed-mouth fruits had the lowest.

Conclusion: This study provided information on the standardization and quality control of evodiamine and rutaecarpine in Evodiae fructus, as well as a foundation for further pharmacological and toxicological studies.

Keywords: Isolation, Quality control, Evodiae Fructus, Major alkaloids

(c) 2019 The Authors. Published by Innovare Academic Sciences Pvt Ltd. This is an open access article under the CC BY license (http://creativecommons.org/licenses/by/4.0/) DOI: http://dx.doi.org/10.22159/ijpps.2019v11i11.34887

\section{INTRODUCTION}

The medicinal plants act as a rich source of natural products. Extraction and characterization of some active components from medicinal plants help in the discovery of new potent drugs [1, 2]. Evodiae fructus (Wuzhuyu in China, ngo-thu-du in Vietnam) is the dried, unripe fruit of Evodia rutaecarpa, which belongs to the family Rutaceae. It has been widely used in Vietnam as a traditional medicine for gastrointestinal disorders and post-partum hemorrhage and amenorrhea. Three of its major components are limonin, evodiamine, and rutaecarpine (fig. 1). Modern pharmacological studies have proved their various activities, such as anti-inflammatory [3, 4], antiobesity [5, 6], hypotensive, cardiotonic, central stimulative, vasodilatory, antithrombotic, and bronchoconstrictive activities $[3,7,8]$.

Interestingly, the degree of maturity of Evodiae fructus is known to influence the content of its active ingredients [9]. Even the fruits from the same plant may have different degrees of maturity, and differ in size, color, shape, and smell. To control the quality of this fruit, the Chinese Pharmacopoeia detects its two major alkaloids, namely evodiamine and rutaecarpine. Several analytical assays for determining evodiamine and rutaecarpine contents have been reported, including liquid chromatography-tandem mass spectrometry (LC/MS/MS) [10,11]. Although the two methods are highly sensitive and selective, their use is limited because of the high cost of their instrumentation. Therefore, this study aimed to develop a simple, rapid, and sensitive analytical method for quantifying biologically important components in Evodiae fructus, namely limonin, evodiamine, and rutaecarpine, to evaluate the quality of Evodiae fructus sold in markets. In this study, 40 batches of Evodiae fructus were collected and the contents of its two major alkaloids were simultaneously determined. Total evodiamine and rutaecarpine contents in different samples were analyzed to provide information on the reasonable use of Evodiae fructus. Open column chromatography was conducted to separate and purify of the two major alkaloids from Evodia rutaecarpa extract possessing the highest evodiamine and rutaecarpine contents.

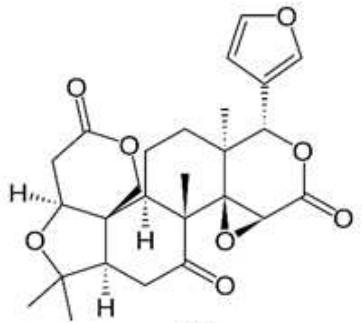

(1)

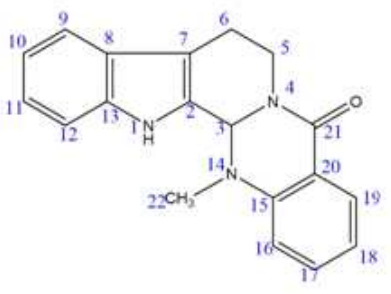

(2)<smiles>O=c1c2ccccc2nc2n1CCc1c-2[nH]c2ccccc12</smiles>

(3)

Fig. 1: Structures of limonin (1), evodiamine (2), and rutaecarpine (3) 


\section{MATERIALS AND METHODS}

\section{Chemical and materials}

HPLC-grade acetonitrile and methanol were purchased from Merck Company (Darmstadt, Germany). Limonin (purity 98.47\%), evodiamine (purity 99\%), and rutaecarpine (purity 99\%), were purchased from Sigma-Aldrich (Milwaukee, WI, USA).

Forty fruits of Evodia rutaecarpa were collected from markets in different regions of Vietnam. The forty voucher specimen of Evodiae fructus was identified at Botany Department of Can Tho University of Medicine and Pharmacy and deposited at the Laboratory of Drug Control and Toxicology. The specimens were stored in sealed packages to avoid exposure to moisture and light.

\section{Preparation of standard}

Standard stock solutions of limonin $(1 \mathrm{mg} / \mathrm{ml})$, evodiamine $(0.2$ $\mathrm{mg} / \mathrm{ml})$, and rutaecarpine $(0.5 \mathrm{mg} / \mathrm{ml})$ were prepared in acetonitrile. The standard working solution of each compound was prepared by diluting the stock solution with a mobile phase to suitable volumes of concentration.

\section{Preparation of sample solutions}

Forty batches of Evodiae fructus were pulverized into a powder, passed through a $0.3 \mathrm{~mm}$ sieve ( 30 meshes), and stored in a desiccator until use. Each powdered sample was accurately weighed $(0.10 \mathrm{~g})$, soaked in $80 \%$ ethanol for $10 \mathrm{~min}$, and then extracted three times with $40 \mathrm{ml} \mathrm{80 \%} \mathrm{ethanol} \mathrm{in} \mathrm{an} \mathrm{ultrasonic} \mathrm{bath} \mathrm{for} 20 \mathrm{~min}$. The extracted solution was filtered through an analytical filter paper and then evaporated to dryness by rotary vaporization under reduced pressure. The residue was suspended in $20 \mathrm{ml}$ of $\mathrm{CH}_{2} \mathrm{Cl}_{2}$ and then successively partitioned twice with water $\left(10 \mathrm{ml}\right.$ each). The $\mathrm{CH}_{2} \mathrm{Cl}_{2}$ extracts were combined and carefully evaporated to dryness in a vacuum. The dried residue was dissolved in $10 \mathrm{ml}$ of mobile phase and injected into the HPLC system for quantitative analysis. All extracts were filtered through a $0.45 \mu \mathrm{m}$ membrane filter into an HPLC vial and capped.

\section{Instrumentation and chromatographic conditions}

The experiment was carried out by a Hitachi HPLC L-2000 system (Hitachi, Japan) equipped with an L-2130 pump, L-2200 syringe, L2300 temperature control system, and L-2455 diode-array detector. Chromatographic separation was achieved using a Germini $\mathrm{C}_{18}$ column $(150 \mathrm{~mm} \times 4.6 \mathrm{~mm}$ I.D., $5 \mu \mathrm{m})$, detected at $210 \mathrm{~nm}$. The mobile phase consisted of acetonitrile (A), methanol (B), and water (C). The gradient elution program was as follows: 0-18 min, linear gradient $40 \% \mathrm{~A}$ and $5 \%$ B with a flow rate of $1 \mathrm{ml} / \mathrm{min} ; 18-30 \mathrm{~min}$, linear gradient $100 \% \mathrm{~A}$ with a flow rate of $1.2 \mathrm{ml} / \mathrm{min}$. The injection volume was $10 \mu \mathrm{l}$.

NMR spectroscopy was performed with a Brucker Advance III (500 $\mathrm{MHz}$; Brucker, Germany) operating at a probe temperature of $23 \pm 1$ ${ }^{\circ} \mathrm{C}$. Tetramethyl silane (TMS) was used as reference $(\delta=0.00 \mathrm{ppm})$. To mix the contents of the NMR tube, a Maxi Mix II mixer (Barnstead/Thermolyne, USA) was used. Spectra were recorded at $500 \mathrm{MHz}$ with $\mathrm{CDCl}_{3}$ (99.8 atom\% D) containing $0.05 \%$ (v/v) TMS as an internal standard.

\section{Method validation}

The proposed method was validated under the guidance of the International Conference on Harmonization of Technical Requirements for the Registration of Pharmaceuticals for Human Use (ICH, 2005) [12]. The assays required to validate the method are as follows: system suitability, selectivity, linearity, limits of detection (LOD), limits of quantification (LOQ), accuracy, and precision assays.

\section{Isolation and purification of two major alkaloids in Evodiae fructus}

\section{Preparation of crude sample}

After alkaloid contents were determined in the 40 batches of Evodiae fructus, fruits possessing the highest alkaloid contents were chosen for extraction, isolation, and purification of evodiamine and rutaecarpine. Each compound was isolated using open column chromatography. Each fraction was manually collected according to the thin layer of the chromatogram and evaporated under reduced pressure. The residues were dissolved in methanol for the subsequent HPLC analysis.

\section{HPLC analysis and identification of each fraction}

The crude extract and all fractions were analyzed by HPLC using a Germini $\mathrm{C}_{18}$ column $(150 \mathrm{~mm} \times 4.6 \mathrm{~mm}$ I.D., $5 \mu \mathrm{m})$. Acetonitrilemethanol-water (15:45:40) was used as a mobile phase in isocratic mode, with a flow rate of $1.0 \mathrm{ml} / \mathrm{min}$. The effluents were monitored at $225 \mathrm{~nm}$ by a photodiode array detector. The identification of each fraction was performed by ${ }^{1} \mathrm{H}$ NMR and ${ }^{13} \mathrm{C}$ NMR. ${ }^{1} \mathrm{H}$ NMR and ${ }^{13} \mathrm{C}$ NMR spectra were recorded on a Bruker Advance III (500 MHz; Brucker, Germany).

\section{Calculation}

According to the Chinese Pharmacopoeia, a high-quality Evodiae fructus should contain at least $0.15 \%$ of total evodiamine and rutaecarpine contents. The total content of each compound was calculated by the following formula:

Content (\%) of analyte $=(C * V * D) / 10000 \mathrm{~W}$

Where:

$\mathrm{C}=$ the concentration $(\mathrm{mg} / \mathrm{l})$ of an analyte in a test solution,

$\mathrm{D}=$ dilution factor, if any,

$\mathrm{V}=$ the final make-up volume $(\mathrm{ml})$ of the test solution,

$\mathrm{W}=$ the weight $(\mathrm{g})$ of the sample used to prepare the test solution.

\section{RESULTS}

\section{Optimization of chromatographic conditions}

Limonin, evodiamine, and rutaecarpine were identified by their retention times $\left(t_{R}\right)$ and by co-injection with standards. The wavelength used to detect limonin, evodiamine, and rutaecarpine in Evodiae fructus was selected by using photodiode-array detection (DAD). The maximum number and height of the three peaks were obtained and the baseline of the chromatogram was stable at 210 $\mathrm{nm}$. Therefore, $210 \mathrm{~nm}$ was chosen as a detection wavelength. The peak purity of the three compounds in the samples was $99.9 \%$, which was the spectrum overlaying the graphs of three-point purity detection. Optimization of chromatographic conditions was performed by using a Gemini RP- $\mathrm{C}_{18}$ with different compositions of mobile phases [methanol-water (1), acetonitrile-water (2), and acetonitrile-methanol-water (3) systems] and a different ratio of solvents in isocratic mode. The results showed that with system 1 , limonin was eluted much more rapidly (2-3 $\mathrm{min})$ than evodiamine and rutaecarpine (50 $\mathrm{min})$.

In contrast, elution with system 2 produced a good resolution of the three components but with short analysis time (under $8 \mathrm{~min}$ ), which proves inconvenient for herbal matrices. Good resolution, baseline, sharp and symmetrical peaks, and favorable retention time were obtained by using system 3 . The mobile phase was acetonitrilemethanol-water in the ratio of 40:5:55, and the retention times of limonin, evodiamine, and rutaecarpine were $8.1,12.5$, and $15.5 \mathrm{~min}$, respectively. However, because of the polar impurity of compounds in the extract solution, we shortened the analysis time by using gradient elution. The representative chromatogram of the sample and standard (fig. 2) showed that limonin, evodiamine, and rutaecarpine were eluted with highly symmetrical peaks under the conditions. The analysis time was $30 \mathrm{~min}$.

\section{Method validation}

\section{System suitability}

System suitability was tested by performing six replicate injections and determining the theoretical plate number (N), resolution (Rs), symmetry factor (As), and repeatability (RSD of retention time and area) of the analyte of interest. The \% RSD values of area and retention time were less than $2 \%$, indicating the precise analysis of limonin, evodiamine, and rutaecarpine by this system. All results showed that the proposed method met the requirements. 


\section{Selectivity}

Method selectivity was tested by using HPLC to compare the retention time of each standard reference compound with that of the peaks of Evodiae fructus extract. The HPLC method was able to distinguish limonin, evodiamine, and rutaecarpine from other constituents in Evodiae fructus (flavonoids, quinolone alkaloids, etc.). There was no interference with the peaks of limonin, evodiamine, and rutaecarpine in Evodiae fructus. Therefore, the peak purity of the three compounds in the sample was $99.9 \%$, as obtained from the spectrum overlaying the graphs of three-point purity detection (fig. 3).
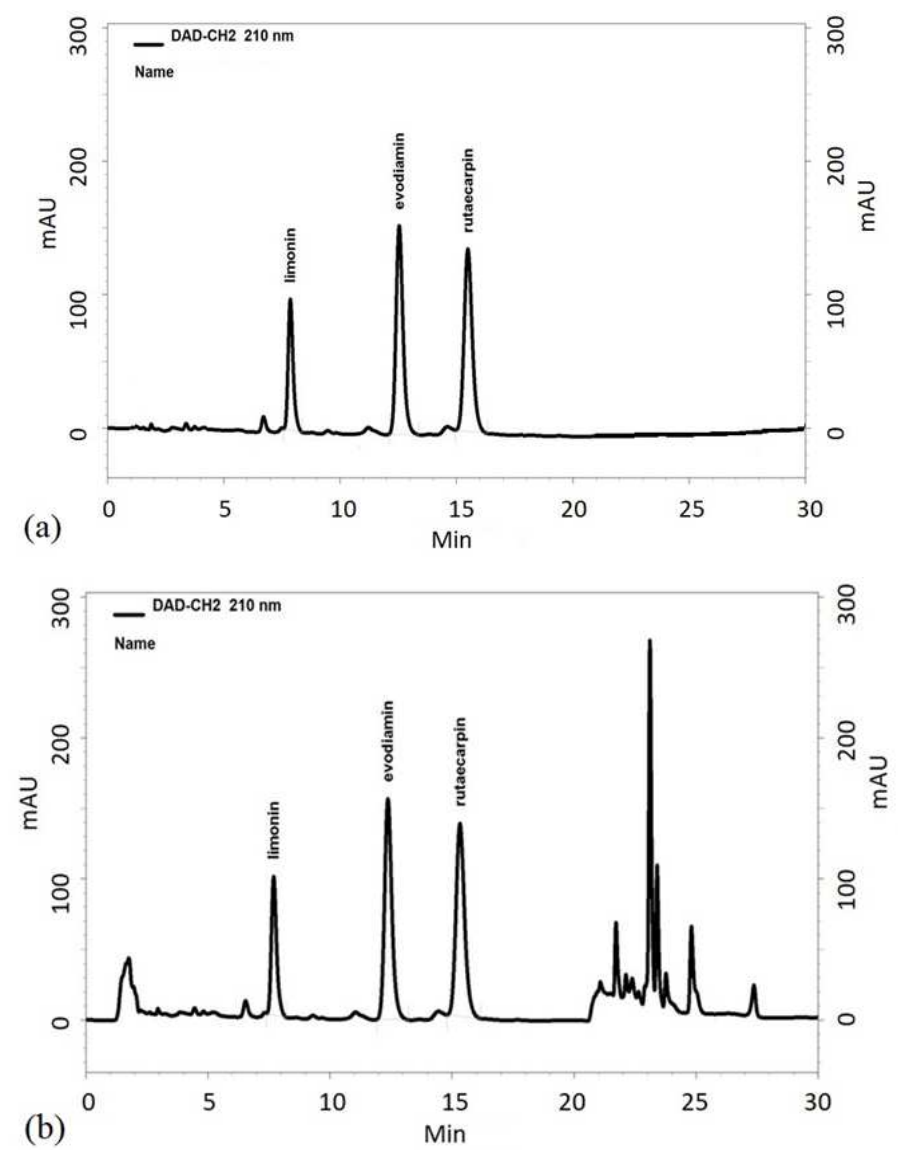

Fig. 2: Representative HPLC chromatograms of mixed standards (a) and Evodiae fructus extract (b) at $210 \mathrm{~nm}$
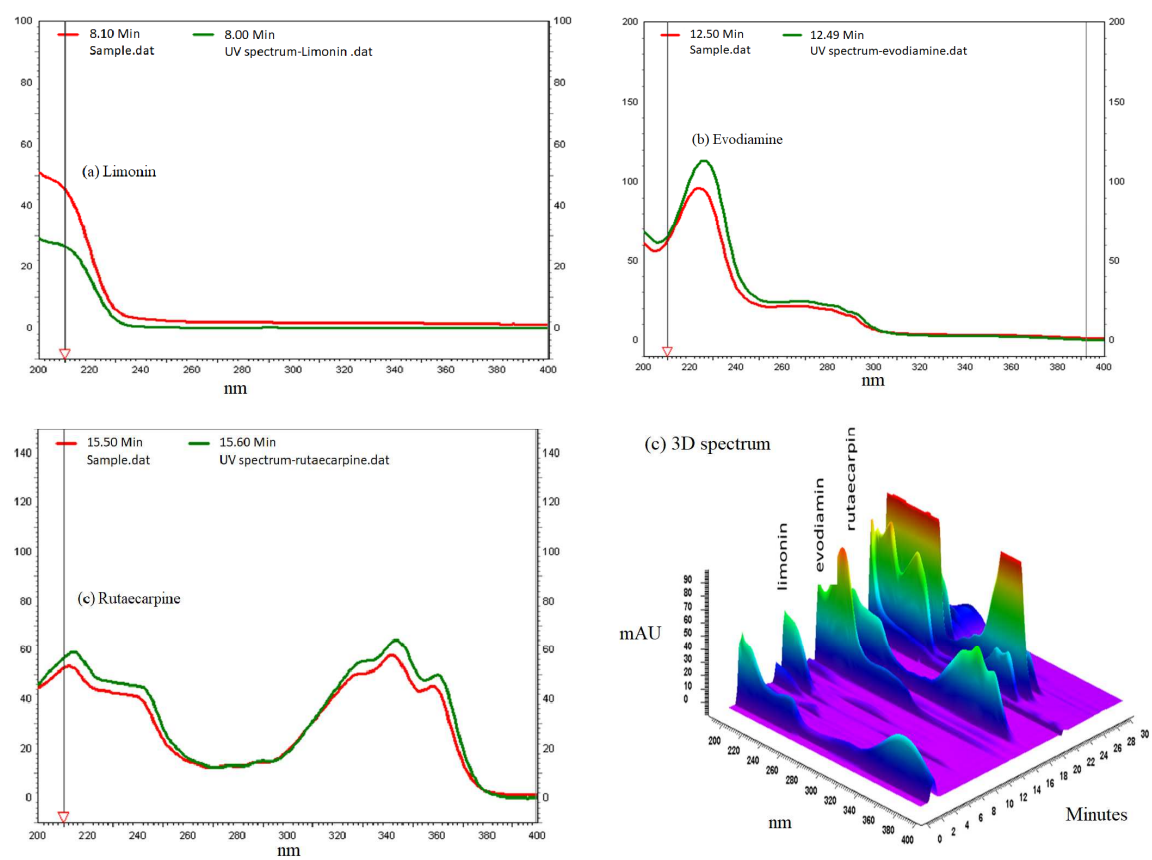

Fig. 3: UV-vis spectrum between standard and extract at their retention time: (a) limonin, (b) evodiamine, (c) rutaecarpine, and (d) 3 D spectrum of Evodiae fructus extraction 
Precision, linearity, limits of detection, and limits of quantification

The results of the regression equation and squared correlation coefficients $\left(\mathrm{r}^{2}\right)$ are summarized in table 1 . The LOD of the three constituents was $0.0625 \mu \mathrm{g} / \mathrm{ml}$. The LOQ of limonin and rutaecarpine was $0.125 \mu \mathrm{g} / \mathrm{ml}$, whereas that of evodiamine was 0.2 $\mu \mathrm{g} / \mathrm{ml}$. The RSDs of intra-day and inter-day were 4.81-6.07, 2.156.79, and $6.01-6.13 \%$ for limonin, evodiamine, and rutaecarpine, respectively.

Table 1: Linear regression data, LOD and LOQ, precision of the HPLC method for determination of limonin, evodiamine and rutaecarpine

\begin{tabular}{|c|c|c|c|}
\hline Parameter & Limonin & Evodiamine & Rutaecarpine \\
\hline Regression equation $^{\mathrm{a}}$ & $y=51262 x-177671$ & $y=268015 x-109314$ & $y=324051 x-875002$ \\
\hline Linearity range $(\mu \mathrm{g} / \mathrm{ml}), \mathrm{n}=8$ & $5-200$ & $5-100$ & $5-200$ \\
\hline 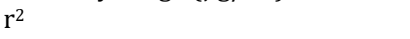 & 0.9977 & 0.9959 & 0.9967 \\
\hline $\mathrm{LOD}(\mu \mathrm{g} / \mathrm{ml}), \mathrm{n}=3$ & 0.0625 & 0.0625 & 0.0625 \\
\hline LOQ $(\mu \mathrm{g} / \mathrm{ml}), \mathrm{n}=3$ & 0.125 & 0.2 & 0.125 \\
\hline Precision (intra-day, $\% \mathrm{RSD}, \mathrm{n}=6$ ) & 4.81 & 2.15 & 6.01 \\
\hline Precision (inter-day, \% RSD, $n=3$ ) & 6.07 & 6.79 & 6.13 \\
\hline
\end{tabular}

$\mathrm{a}=\mathrm{y}$ is the concentration of the analyte $(\mu \mathrm{g} / \mathrm{ml})$, and $\mathrm{x}$ is the peak area, $\mathrm{n}=$ number of determination, $\%$ RSD $=\%$ Relative standard deviation

\section{Accuracy}

Table 2 shows a summary of extraction recovery in Evodiae fructus samples. The developed method had good accuracy with an overall recovery of $102.73,101.58$, and $103.34 \%$ for limonin, evodiamine, and rutaecarpine, respectively, with \% RSD of less than $5 \%$ for the analytes. Considering the results of the recovery test, the method was deemed to be accurate.

Table 2: Recoveries for the assay of the investigated compounds in Evodiae fructus

\begin{tabular}{|c|c|c|c|c|c|c|c|}
\hline \multirow[t]{2}{*}{ Analytes } & \multirow[t]{2}{*}{ Sample } & \multicolumn{3}{|c|}{ Concentration $(\mu \mathrm{g} / \mathrm{ml})$} & Recovery & \multirow{2}{*}{$\begin{array}{l}\text { Mean recovery } \\
n=9\end{array}$} & \multirow{2}{*}{$\begin{array}{l}\text { RSD (\%) } \\
n=9\end{array}$} \\
\hline & & Original & Added & Found & $(\%)$ & & \\
\hline \multirow[t]{4}{*}{ Limonin } & $\mathrm{S}_{1}^{\mathrm{a}}$ & 56.11 & 50 & 106.68 & 101.14 & & \\
\hline & $\mathrm{S}_{2} \mathrm{~b}$ & 56.11 & 60 & 118.36 & 103.76 & $102.73 \pm 5.12$ & 4.98 \\
\hline & $\mathrm{S}_{3} \mathrm{c}$ & 56.11 & 70 & 128.42 & 103.30 & & \\
\hline & $\mathrm{S}_{1}^{\mathrm{a}}$ & 19.87 & 15 & 34.88 & 100.06 & & \\
\hline \multirow[t]{3}{*}{ Evodiamine } & $\mathrm{S}_{2} \mathrm{~b}$ & 19.87 & 20 & 39.86 & 99.94 & $101.58 \pm 4.51$ & 4.44 \\
\hline & $\mathrm{S}_{3} \mathrm{c}$ & 19.87 & 25 & 46.05 & 104.73 & & \\
\hline & $\mathrm{S}_{1}^{\mathrm{a}}$ & 20.64 & 15 & 36.01 & 102.46 & & \\
\hline \multirow[t]{2}{*}{ Rutaecarpine } & $\mathrm{S}_{2} \mathrm{~b}$ & 20.64 & 20 & 41.09 & 102.27 & $103.34 \pm 3.91$ & 3.78 \\
\hline & $\mathrm{S}_{3} \mathrm{c}$ & 20.64 & 25 & 46.96 & 105.30 & & \\
\hline
\end{tabular}

Recovery $(\%)=(($ found-original $) /$ added $) \times 100$., aThe samples added known amounts of standards at low level $(80 \%$ of the known amounts $)$., ${ }^{b}$ The samples added known amounts of standards at medium level (same as the known amounts)., "The samples added known amounts of standards at high level (120\% of the known amounts), $\%$ RSD $=\%$ Relative standard deviation

\section{Quality evaluation of Evodiae fructus in markets}

Evodiamine and rutaecarpine contents in 40 samples of Evodiae fructus are summarized in table 3 and 4 . Data are expressed as \% (grams per 100 gram) of dry weight. The results showed a relationship between alkaloid contents and fruit size (fig. 4). The contents of the two major alkaloids were also significantly correlated with maturity degrees, as shown in fig. 5.

Table 3: Correlations between alkaloid contents and fruit size

\begin{tabular}{llll}
\hline Fruit size (mm) & N & Mean of alkaloids content (\%) & Test \\
\hline 2 & 9 & 0.1744 & Spearman parameter r=0.597 \\
3 & 9 & 0.2024 & p<0.001 \\
4 & 3 & 0.4179 & \\
5 & 6 & 0.9539 & \\
6 & 8 & 0.7214 & \\
7 & 3 & 0.3624 & \\
8 & 2 & 0.3124 & \\
Total & 40 & 0.44629 & \\
\hline
\end{tabular}

$N=$ number of samples

Table 4: Correlations between alkaloid contents and maturity degree

\begin{tabular}{lllll}
\hline Maturity degree & $\mathbf{N}$ & Content & Kruskal wallis test $\chi^{2} \cdot \mathbf{p}$ \\
\cline { 2 - 5 } & & mean \pm SD & 0.1445 & $\chi^{2}=11.1$ \\
Closed mouth fruit & 12 & $0.18716 \pm 1156.4$ & 0.3875 & $\mathrm{p}=0.004$ \\
Open-mouth fruit & 19 & $0.65173 \pm 7075.9$ & 0.2892 & \\
Large mouth fruit & 9 & $0.3581 \pm 2036.7$ & 3013 & \\
Total & 40 & $0.44629 \pm 5351.4$ & \\
\hline
\end{tabular}

$\mathrm{N}=$ number of samples, $\mathrm{SD}=$ Standard deviation 


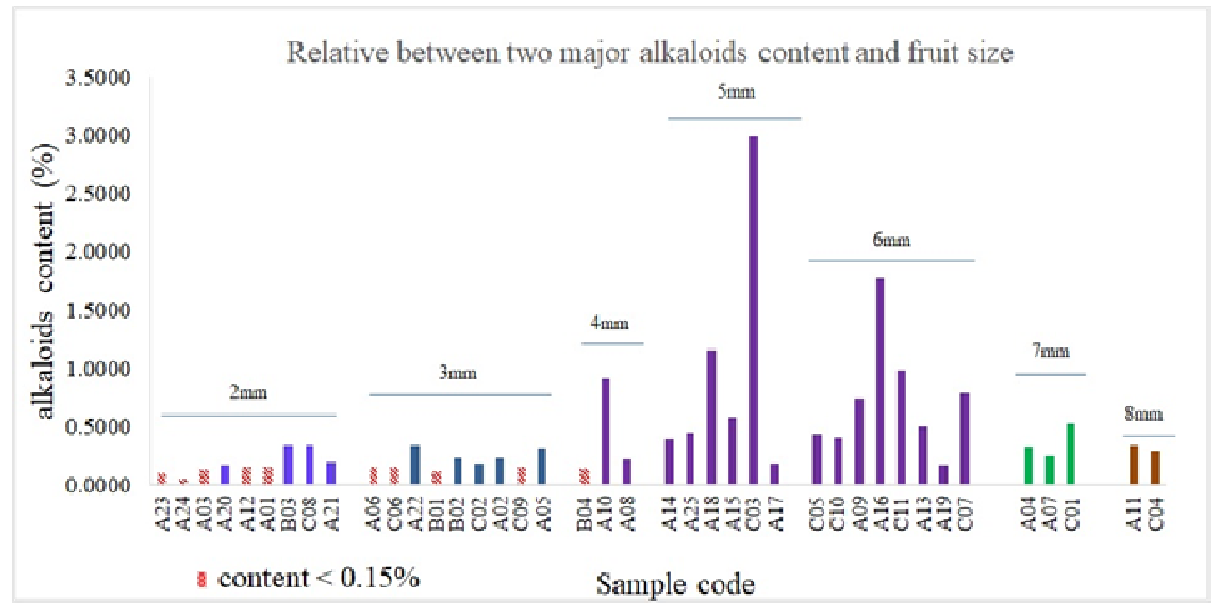

Fig. 4: Correlation of alkaloids content of Evodiae fructus samples and their fruit size

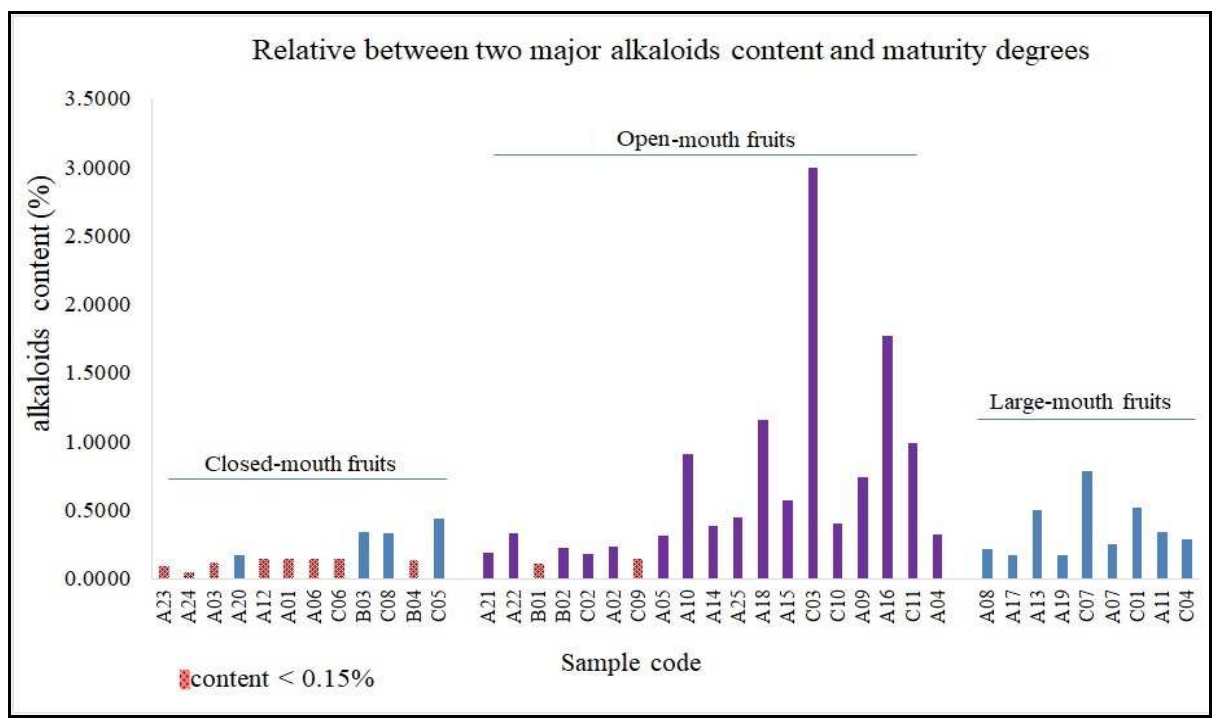

Fig. 5: Correlation of alkaloids content of Evodiae fructus samples and their maturity degrees

\section{Isolation and purification of rutaecarpine and evodiamine}

\section{Open column chromatography and purification conditions}

A series of experiments were performed to optimize the mobile phase solvent system for the proposed column separation method. Chloroform-methanol, chloroform-ethyl acetate, and $n$-hexane-ethyl acetate were tested as mobile phase solvents. When ethyl acetatemethanol was used, the target compounds mainly co-elute. By using chloroform-ethyl acetate, the elution was improved, but target compounds and nonpolar impurities were not separated. Thus, chloroform-methanol and chloroform-ethyl acetate were unsuitable to separate and purify the alkaloids in Evodiae fructus. In contrast, a mixture of $n$-hexane and ethyl acetate improved the fraction separation. The first fraction was purified by washing with cool n-hexane and a pure second fraction was obtained through precipitation with chloroform.

Two kinds of alkaloids were obtained in the one-step purification, yielding $326 \mathrm{mg}$ rutaecarpine (I) and $128 \mathrm{mg}$ of evodiamine (II) from 3.2 g crude sample. The purities of the two compounds were 98.9 and $98.5 \%$ respectively, as determined by HPLC. The chromatograms and UV spectra of these compounds are shown in fig. 6.

\section{The structural identification}

The chemical structure of each fraction was identified according to its ${ }^{1} \mathrm{H}$ NMR, ${ }^{13} \mathrm{C}$ NMR and ESI-MS data:
Fraction (I): ${ }^{1} \mathrm{H}-\mathrm{NMR}\left(500 \mathrm{MHz}, \mathrm{CDCl}_{3}\right) \delta$ ppm: 9.51 (br s. $\left.1 \mathrm{H} . \mathrm{NH}\right)$; 8.32 (d. 1H; J=7; H19); 7.70 (m. 1H. H17); 7.63 (m. 1H; H16); 7.42 (m. 1H; H9); 7.36 (m. 1H; H12); 7.31 (m. 1H; H18); 7.25 (s. 1H; H11); 7.17 (m. 1H; H10); 4.59 (t. 2H; H5); 3.23 (t. 2H; H6). ${ }^{13} \mathrm{C}-$ NMR $\left(125 \mathrm{MHz}, \mathrm{CDCl}_{3}\right) \delta$ ppm: 127.2 (C-2), 145.4 (C-3), 41.1 (C-5), 19.7 (C-6), 118.4 (C-7), 125.6 (C-8), 120.6 (C-9), 120.1 (C-10), 125.6 (C-11), 112.1 (C-12), 138.3 (C-13), 147.5 (C-15), 126.2 (C16), 134.3 (C-17), 127.1 (C-18), 126.6 (C-19), 121.1 (C-20), 162.0 (C-21). ESI-MS m/z 288.1131 calculated for $\mathrm{C}_{18} \mathrm{H}_{13} \mathrm{~N}_{3} \mathrm{O}$. According to the data obtained by Liu et al., fraction I corresponded to rutaecarpine [14].

Fraction (I): ${ }^{1} \mathrm{H}-\mathrm{NMR}\left(500 \mathrm{MHz}, \mathrm{CDCl}_{3}\right) \delta p p m: 8,24(\mathrm{br} s, 1 \mathrm{H}$, $\mathrm{NH}) ; 8,12\left(d d, 1 \mathrm{H} ; J=6.5 ; \mathrm{H}_{19}\right) ; 7,59(d, 1 \mathrm{H} ; J=7.5 ; \mathrm{H} 9) ; 7,49(m, 1 \mathrm{H}$, $\left.\mathrm{H}_{17}\right) ; 7,41\left(d, 1 \mathrm{H}, J=8 ; \mathrm{H}_{12}\right) ; 7,25\left(m, 2 \mathrm{H} ; \mathrm{H}_{11}, \mathrm{H}_{16}\right) ; 7,19(m, 2 \mathrm{H}$; $\left.\mathrm{H}_{10}, \mathrm{H}_{18}\right) ; 5,92\left(s, 1 \mathrm{H} ; \mathrm{H}_{3}\right) ; 4,87\left(m, 1 \mathrm{H} ; \mathrm{H}_{5}\right) ; 3,29\left(m, 2 \mathrm{H} ; \mathrm{H}_{5}\right) ; 2,96$ $\left(m, 2 \mathrm{H} ; \mathrm{H}_{6}\right) ; 2,505\left(s, 3 \mathrm{H} ; \mathrm{N}-\mathrm{CH}_{3}\right) .{ }^{13} \mathrm{C}-\mathrm{NMR}\left(125 \mathrm{MHz}, \mathrm{CDCl}_{3}\right) \delta$ ppm: 129.0 (C-2), 68.8 (C-3), 39.5 (C-5), 20.1 (C-6), 113.7 (C-7), 126.3 (C-8), 118.9 (C-9), 123.1 (C-10), 124.1 (C-11), 111.3 (C12), 136.7 (C-13), 150.6 (C-15), 122.4 (C-16), 133.1 (C-17), 123.8 (C-18), 128.2 (C-19), 120.0 (C-20), 164.7 (C-21), 37.2 (N CH3). ESI-MS m/z 303.1464 calculated for $\mathrm{C}_{19} \mathrm{H}_{17} \mathrm{~N}_{3} \mathrm{O}$. In accordance with the data obtained by Liu et al., fraction II corresponded to evodiamine [14]. 


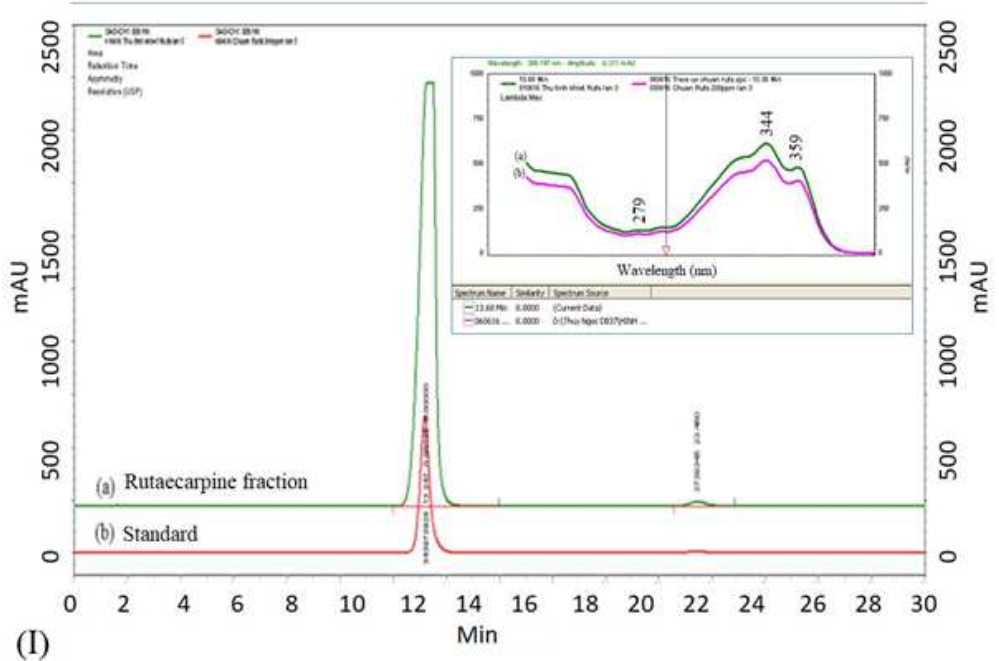

(I)

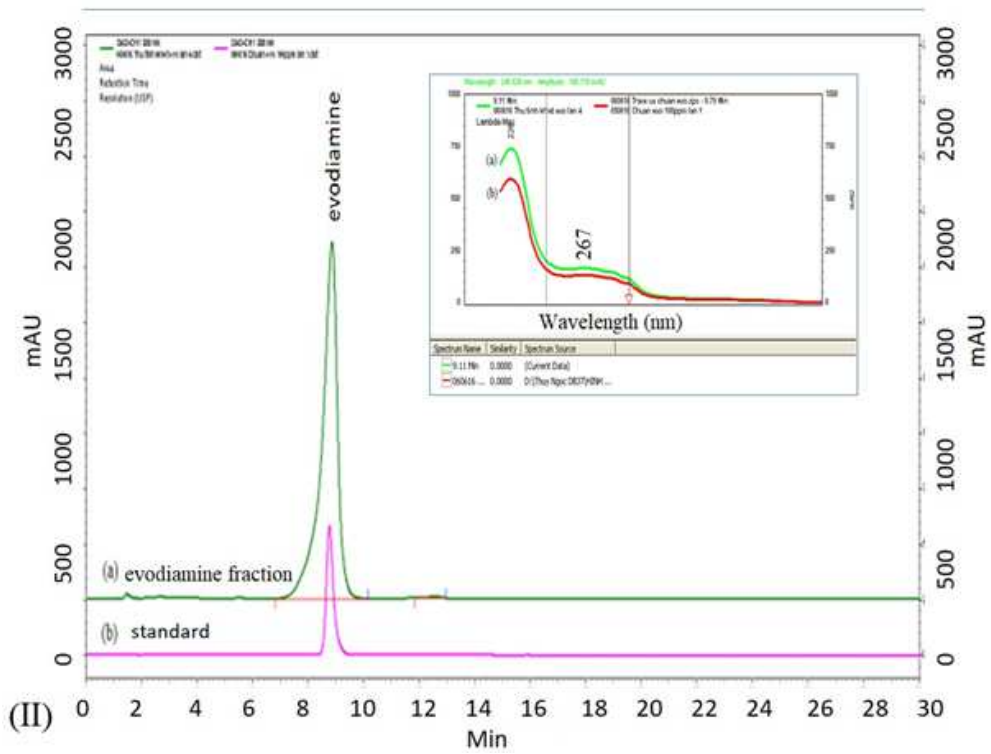

Fig. 6: HPLC purity test of two fractions collected from Evodiae fructus extraction, fraction (I): rutaecarpine, fraction (II) evodiamine

\section{DISCUSSION}

Contents of the three major components in Evodiae fructus extract could easily be determined within $30 \mathrm{~min}$. All calibration curves showed good linear regression $\left(\mathrm{r}^{2}>0.99\right)$, with RSDs of intra-day and inter-day of approximately $6 \%$. The overall recovery of the analytes was in the range of 101.58 to $103.34 \%$ with \% RSD of less than $5 \%$; thus, the proposed method was deemed to be precise and accurate. Zhao et al. developed an LC method for determining dehydroevodiamine, wuchuyuamide-I, 5-hydroxyrutaecarpine, 14formyldihydrorutaecarpine, evodiamine, and rutaecarpine contents; however, the method requires a long analysis time of $70 \mathrm{~min}$ and complicated mobile phase consisting of methanol, acetonitrile, and phosphoric acid-triethylamine-buffer solution [13]. In this study, we conducted a simple chromatography using popular solvents commonly available in laboratories, which required only a short time $(30 \mathrm{~min})$ to measure the amount of the three major components in Evodiae fructus.

The validated method was successfully applied to simultaneously determine the contents of the two major alkaloids of Evodiae fructus in 40 samples collected from markets in different regions of Vietnam. As shown in table 3, alkaloid concentrations were the highest in the open-mouth fruits, followed by those in the largemouth fruits and closed-mouth fruits, with $\chi^{2}=11.1$ and $p=0.004$, as analyzed by the Kruskal-Wallis test. Closed-mouth fruits showed low contents of the two major alkaloids. In this group, 8 out of 12 samples showed evodiamine and rutaecarpine contents of less than $0.15 \%$. In contrast, in open-mouth fruits, 19 samples showed high evodiamine and rutaecarpine contents. For instance, sample C03 showed total evodiamine and rutaecarpine contents of up to $3 \%$. Interestingly, in large mouth-fruits (similar to open-mouth fruits except that their ovaries are split completely into five compartments), 9 samples showed uniformly high evodiamine and rutaecarpine contents. Similarly, as shown in table 4 and fig. 6 , the highest alkaloid concentrations were observed in the fruit group with an average size of 4 to $6 \mathrm{~mm}$, followed by those with big sizes of 7 to $8 \mathrm{~mm}$ and small sizes of 1 to $3 \mathrm{~mm}$. The differences were significant, with a Spearman correlation coefficient of $r>0.5$ and $\mathrm{p}<0.001$. The results were useful as a guide for choosing Evodiae fructus base on the fruit's degree of maturity.

In a one-step purification of $3.2 \mathrm{~g}$ crude extract, we obtained 326 and $128 \mathrm{mg}$ of rutaecarpine (98.9\% purity) and evodiamine $(98.5 \%$ purity), respectively. Liu et al. used high-speed counter-current chromatography with a two-phase solvent system to isolate and purify five alkaloids, including $18 \mathrm{mg}$ of evodiamine and $9 \mathrm{mg}$ of rutaecarpine (purity of $98.4 \%$ ), from 6.2 g crude sample [14]. In comparison with our results, we can conclude that our method was effective and economical. It can be implied that these pure alkaloids can be used as a standard to control the quality of Evodiae fructus in Vietnam. Currently, the Vietnamese Pharmacopoeia uses total oil 
content to evaluate the quality of this fruit. Our results provided an option to use natural standard compounds, namely evodiamine and rutaecarpine, to control the quality of Evodiae fructus in the market.

\section{CONCLUSION}

In summary, this study described the development, validation, and application of an HPLC method for determining limonin, evodiamine, and rutaecarpine contents in Evodiae fructus using Germini $\mathrm{C}_{18}$ column. The relatively simple sample preparation, together with the short HPLC run time (30 min), proved that the present method was useful for routine quantitative analysis and quality control of limonin, evodiamine, and rutaecarpine, the major components in Evodiae fructus. This method was satisfactory in terms of accuracy, precision, sensitivity, and reproducibility. The method was successfully applied to analyze 40 batches of samples collected from markets, and the results showed that alkaloid contents were related to fruit size and fruit maturity degree. The highest alkaloid concentrations were observed in fruits with an average size of 4 to 6 $\mathrm{mm}$, and open-mouth fruits. The proposed method was shown to be useful as a guide for choosing Evodiae fructus base on its maturity degree. Next, fruits possessing the highest alkaloid contents were chosen for isolation and yielded $326 \mathrm{mg}$ of rutaecarpine and $128 \mathrm{mg}$ of evodiamine from 3.2 g crude sample. The purity was 98.9 and $98.5 \%$, respectively, as determined by HPLC. This study sheds light on the standardization and quality control of evodiamine and rutaecarpine.

\section{ACKNOWLEDGMENT}

The authors thank the Can Tho University of Medicine and Pharmacy for the use of analytical instruments. We also thank all of our colleagues for their excellent assistance.

\section{AUTHORS CONTRIBUTIONS}

N. Van. T. Nguyen, X. Dao T. Nguyenperformed the experiments, K. Ngan H. Nguyen and T. Kien Nguyen analyzed the data; N. Van. T. Nguyen and K. Ngan H. Nguyen wrote and edited the paper; M. Phuong Nguyen gave suggestion, Kyeong-Ho Kim supervised the project.

\section{CONFLICT OF INTERESTS}

The authors declare that there is no conflict of interest

\section{REFERENCES}

1. Popova A, Mihaylova D. A review of the medicinal plants in Bulgaria-collection, storage, and extraction techniques. Asian J Pharm Clin Res 2018;11:28-35.

2. Shelar M, Sathiyanarayanan L, Mahadik K, Gadgil S. Standardization and comparative evaluation of ayurvedic polyherbal ghrita formulation with modern extraction technique for extraction efficiency using reversed phase-high performance liquid chromatography. Asian J Pharm Clin Res 2018;10:264-71.

3. Liao JF, Chiou WF, Shen YC, Wang GJ, Chen CF. Antiinflammatory and anti-infectious effects of Evodia rutaecarpa (Wuzhuyu) and its major bioactive components. Chin Med 2011;6:1-8.

4. Choi YH, Shin EM, Kim YS, Cai XF, Lee JJ, Kim HP. Antiinflammatory principles from the fruits of Evodia rutaecarpa and their cellular action mechanisms. Arch Pharm Res 2006;29:293-7.

5. Wang T, Wang Y, Kontani Y, Kobayashi Y, Sato Y, Mori N, et al. Evodiamine improves diet-induced obesity in a uncoupling protein-1-independent manner: involvement of antiadipogenic mechanism and extracellularly regulated kinase/mitogenactivated protein kinase signaling. Endocrinology 2008; 149:358-66.

6. Kobayashi Y, Nakano Y, Kizaki M, Hoshikuma K, Yokoo Y, Kamiya T. Capsaicin-like anti-obese activities of evodiamine from fruits of Evodia rutaecarpa, a vanilloid receptor agonist. Planta Med 2001;67:628-33.

7. Zhou X, Zhao Y, Lei P, Cai Z, Liu H. Chromatographic fingerprint study on Evodia rutaecarpa (Juss.) benth by HPLC/DAD/ESI$M^{n}$ technique. J Sep Sci 2010;33:2258-65.

8. Lee SH, Son JK, Jeong BS, Jeong TC, Chang HW, Lee ES, et al. Review: progress in the studies on rutaecarpine. Molecules 2008;13:272-300.

9. Pellati F, Benvenuti S, Yoshizaki F, Melegari M. Development and validation of HPLC methods for the analysis of phenethylamine and indoloquinazoline alkaloids in evodia species. J Sep Sci 2006;29:641-9.

10. Zhao Y, Zhao Y, Zhou X, Gong X. Development and validation of an UPLC-ESI-MS/MS method for determination of dehydroevodiamin, limonin, evodiamine and rutaecarpine in Evodiae Fructus. Pharmacogn Mag 2014;10:374-83.

11. Lin C, Pan X, Li W, Ma J, Pan J, Cai J, et al. Simultaneous determination of evodiamine and rutecarpine in rabbit plasma by LC-ESI-MS and its application to pharmacokinetics. Pharmazie 2011;66:920-3.

12. ICH Harmonised Tripartite Guideline. International Conference on Harmonisation of Technical Requirements for Registration of Pharmaceuticals for Human Use. Validation of Analytical Procedure Text and Methodology Q2 (R1); 2005.

13. Zhao MY, Yang XW. Optimization of the extraction conditions and simultaneous quantification by RP-LC of six alkaloids in Evodiae fructus. Chromatographia 2008;67:543-50.

14. Liu R, Chu X, Sun A, Kong L. Preparative isolation and purification of alkaloids from the Chinese medicinal herb Evodia rutaecarpa (Juss.) Benth by high-speed counter-current chromatography. J Chromatogr 2005;1074:139-44. 